\title{
Electrogene therapy with interleukin-12 in canine mast cell tumors
}

\author{
Darja Pavlin'1, Maja Cemazar ${ }^{2,3}$, Andrej Cör ${ }^{3}$, Gregor Sersa², Azra Pogacnik¹, Natasa Tozon¹ \\ 1 University of Ljubljana, Veterinary Faculty Ljubljana, Ljubljana, Slovenia \\ ${ }^{2}$ Institute of Oncology Ljubljana, Department of Experimental Oncology, Ljubljana, Slovenia \\ ${ }^{3}$ University of Primorska, College of Health Care Izola, Izola, Slovenia
}

Received 29 June 2010

Accepted 29 August 2010

Correspondence to: Prof. dr. Natasa Tozon, University of Ljubljana, Veterinary Faculty Ljubljana, Gerbičeva 60, SI-1000 Ljubljana, Slovenia. E-mail: natasa.tozon@vf.uni-lj.si

Disclosure: No potential conflicts of interest were disclosed.

Background. Mast cell tumors (MCT) are the most common malignant cutaneous tumors in dogs with extremely variable biological behaviour. Different treatment approaches can be used in canine cutaneous MCT, with surgical excision being the treatment of choice. In this study, electrogene therapy (EGT) as a new therapeutic approach to canine MCTs, was established.

Materials and methods. Eight dogs with a total of eleven cutaneous MCTs were treated with intratumoral EGT using DNA plasmid encoding human interleukin-12 (IL-12). The local response to the therapy was evaluated by repeated measurements of tumor size and histological examination of treated tumors. A possible systemic response was assessed by determination of IL-12 and interferon- $Y$ (IFN-Y) in patients' sera. The occurence of side effects was monitored with weekly clinical examinations of treated animals and by performing basic bloodwork, consisting of the complete bloodcount and determination of selected biochemistry parameters.

Results. Intratumoral EGT with IL-12 elicits significant reduction of treated tumors' size, ranging from 13\% to $83 \%$ (median $50 \%$ ) of the initial tumor volume. Additionally, a change in the histological structure of treated nodules was seen. There was a reduction in number of malignant mast cells and inflammatory cell infiltration of treated tumors. Systemic release of IL-12 in four patients was detected, without any noticeable local or systemic side effects.

Conclusions. These data suggest that intratumoral EGT with plasmid encoding IL-12 may be useful in the treatment of canine MCTs, exerting a local antitumor effect.

Key words: electroporation; electrotransfection; electrogene therapy; mast cell tumors; IL-12; IFN-Y

\section{Introduction}

Mast cell tumors (MCT) are the most common malignant cutaneous tumors in dogs, accounting for around $21 \%$ of all cutaneous tumors. ${ }^{1}$ Cutaneous MCT have extremely variable biological behavior, from low-grade tumors to highly invasive lesions with high metastatic potential, which makes proper staging and treatment of MCT very challenging.

Treatment options for canine MCT depend on prognostic factors, primarily the histological grade of the tumor and clinical stage of the disease. The treatment of choice for MCT is wide surgical excision, when possible, which results in excellent prognosis for well-differentiated MCT. ${ }^{1}$ Poorly differentiated or anaplastic MCT carry a poor prognosis and in these tumors aggressive surgical treatment should be followed by other treatment modalities, e.g. radio- or chemotherapy. ${ }^{1}$ In dogs, where it is not possible to perform surgical excision and in cases with advanced stages of disease, systemic chemotherapy is the most appropriate treatment option..$^{1,2}$

One of the newer therapeutic approaches for local tumor control is electrochemotherapy (ECT), which has already been established as a successful treatment option for different histological types of canine tumors, including MCT. ${ }^{3}$ It employs intralesional or systemic injection of the chemotherapeutic agent bleomycin or cisplatin, followed by local delivery of electric pulses to the tumor nodule, which significantly increase uptake and cytotox- 
icity of chemotherapeutic drugs. ${ }^{4-6}$ The procedure is based on electroporation of the cell membrane, achieving a transient increase in its permeability, thus allowing intracellular uptake of chemotherapeutic drugs from the extracellular space. ${ }^{7}$

The same principle can also be used for intracellular delivery of other molecules, for example plasmid DNA. Combining direct injection of plasmid DNA containing a therapeutic gene into target tissue, together with local delivery of electric pulses is called electrogene therapy (EGT) ${ }^{8-10}$ In veterinary medicine, it has already been used for delivery of different transgenes into skeletal muscle ${ }^{11-13}$ and intratumorally. ${ }^{14,15}$

IL-12 exhibits a range of biological activities, potentially important in immunotherapy of cancer. These include, for example, activation of natural killer cells, induction of IFN- $\gamma$, inhibition of angiogenesis and stimulation of nitric oxide production. ${ }^{16}$ Gene therapy using $I L-12$ has already shown remarkable antitumor activity in different tumor models at the preclinical level, and has already progressed to a number of clinical trials in both human and veterinary medicine. ${ }^{17-21}$

The aim of our study was to evaluate the local antitumor effect, systemic transgene release and possible side effects of EGT with the therapeutic plasmid encoding human IL-12 in canine MCT. For this purpose, a plasmid encoding human IL-12 was injected intratumorally into spontaneously occurring superficial nodules of MCT in 8 patients, followed by application of electric pulses. Local response to therapy was evaluated by regular measurements of tumor size and histological assessment of excised tumor nodules. Systemic transgene release was determined by measurements of IL-12 and IFN- $\gamma$ in patients' sera. Possible side effects of the procedure were monitored by regular determination of selected hematology and biochemistry parameters.

\section{Materials and methods}

\section{Animals}

All animals in this study were referred to the Veterinary Faculty of Ljubljana in February and March 2006 for evaluation of cutaneous or subcutaneous tumor nodules. Eight patients that corresponded to inclusion criteria for the clinical study were included. The study cohort was comprised of 3 intact males and 5 spayed females of 6 different breeds ( 3 German boxers, and one of each: crossbreed, toy poodle, French bulldog, Siberian husky and bullterrier), their age ranging from 5 to 16 years (Table 1). Inclusion criteria for the study comprised at least one cytologically or histologically confirmed MCT, good general health status of the animal with the basic hematology and biochemistry profile within reference limits and normal renal and cardiovascular function. Animals included in the study were either ones which were planned for surgical excision of the tumor nodule as a part of standard therapeutic procedure and their owners agreed to the EGT procedure prior to surgery, or had recurrent disease in which other conventional therapy methods were already exhausted by previous treatments, or their owners refused any other type of standard treatment at the time of inclusion. Prior to inclusion, written consent for participation in the clinical study for each animal was obtained from their owners. The study was approved by the Ethical Committee at the Ministry of Agriculture, Forestry and Food of the Republic of Slovenia (approval No. 323-451/2004-9).

Clinical examination of each animal was performed before the treatment. Fine needle aspiration biopsy of tumor nodules, as well as of local lymph nodes, was taken and cytological examination of samples was performed. In all animals, staging was performed according to modified WHO staging criteria ${ }^{22}$ with examination of thoracic radiographs, abdominal ultrasonography and basic bloodwork. Basic bloodwork consisted of a complete blood count with differential white blood cell count, which was performed using an automated laser hematology analyzer (Technicon $\mathrm{H}^{*} 1$, Bayer, Germany) with species-specific software $\left(\mathrm{H}^{*} 1\right.$ Multi-Species V30 Software). The automated chemistry analyzer Technicon RA-XT (Bayer, Germany) was used for determination of the following biochemical parameters: blood urea nitrogen (BUN), creatinine, serum alkaline phosphatase (SAP) and alanine aminotransferase (ALT). Additionally, serum concentrations of human IL-12 and canine IFN- $\gamma$ were determined using ELISA kits (Human IL-12 Quantikine ELISA Kit and Canine IFN- $\gamma$ Quantikine ELISA Kit, respectively, both R\&D Systems, Minneapolis, MN, USA).

A total of 11 tumor nodules were treated with EGT in the 8 patients which were included in the study. Two dogs received only one EGT, four dogs received 2 EGTs, with the second EGT session delivered one week after the first treatment, and one dog received 3 EGT sessions, each one week apart. In the remaining $d o g$, four sessions were performed, each one month apart (Table 2).

In the patients, EGT was performed either as a single therapy ( $2 / 8$ patients) or it was followed 

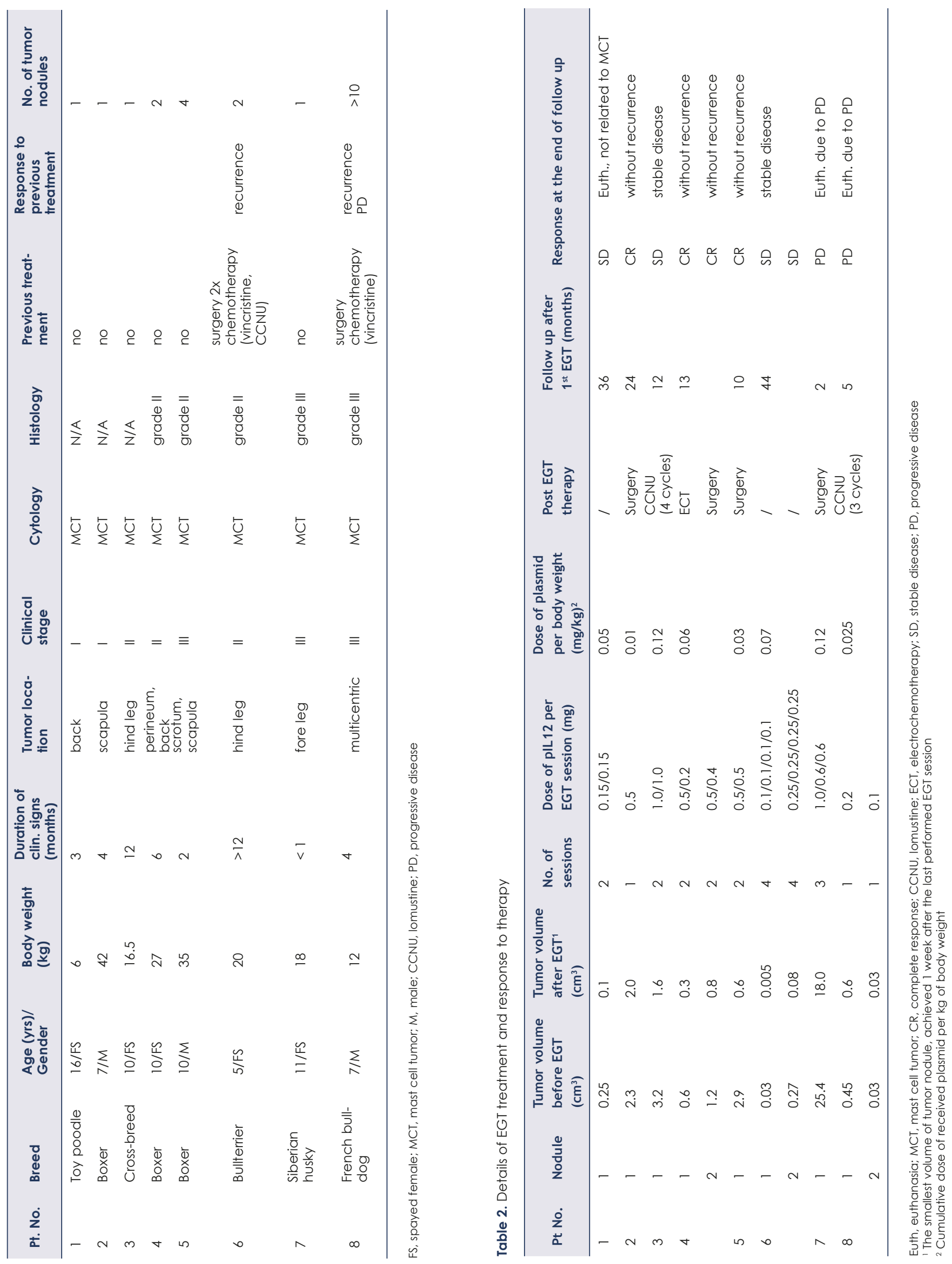

Radiol Oncol 2011 ; $45(1)$ : 30-39. 
with one of the following therapies: standard surgical removal of MCT nodules (4/8 patients), chemotherapy (2/8 patients) or ECT (one nodule in a patient with two MCTs) (Table 2). Treated tumors were surgically removed one week after the last EGT session. In patients with systemic chemotherapy, 3 and 4 cycles of CCNU (lomustine, 60$90 \mathrm{mg} / \mathrm{m}^{2}$, every 3 weeks) were delivered, starting two weeks after the last EGT session. ECT was performed one week after the last EGT session with intratumoral application of cisplatin (Cisplatyl, Aventis, Paris, France) at a dose $1 \mathrm{mg} / \mathrm{cm}^{3}$ of tumor tissue, followed by application of electric pulses $(8$ pulses of $100 \mu$ s duration at an amplitude of 1300 $\mathrm{V} / \mathrm{cm}$ and frequency $1 \mathrm{~Hz}$ ), using the same electric pulse generator as described below.

In animals in which histological samples of tumors were obtained either as part of the diagnostic procedure or with surgical excision after EGT, tumor samples were histologically evaluated. Histological grading was established using Patnaik's histological criteria. ${ }^{22}$

\section{Plasmid preparation}

The pORF-hIL-12 plasmid (InvivoGen, Toulouse, France), encoding human IL-12, was selected according to available data indicating that canine and human IL-12 share approximately $90 \%$ genetic identity based on amino acid sequence analysis. ${ }^{23}$ It has already been shown that human IL-12 activates proliferation of canine peripheral blood mononuclear cells (PBMC) in an in vitro setting and triggers a number of immune responses in canine PBMC. ${ }^{24}$ The plasmid was prepared using the Qiagen Endo-Free kit (Qiagen, Hilden, Germany), according to the manufacturer's instructions and diluted to a concentration of $1 \mathrm{mg} / \mathrm{ml}$. Purified plasmid DNA was subjected to quality control and quantity determinations, performed by agarose gel electrophoresis and by means of spectrophotometry.

\section{Electrogene therapy procedure}

EGT was performed in animals under general anesthesia, which was induced with propofol (Propoven $10 \mathrm{mg} / \mathrm{ml}$, Fresenius Kabi Austria GmbH, Graz, Austria) and maintained with isoflurane (Forane, Abbott Laboratories LTD, Queensborough, United Kingdom). During the anesthesia, animals received Harmann's solution (B. Braun Melsungen AG, Melsungen, Germany) at a rate of $10 \mathrm{ml} / \mathrm{kg}$ of body weight $/ \mathrm{h}$.
In animals under general anesthesia, hair overlying tumor nodules was removed, carefully avoiding any unnecessary manipulation of tumors which could lead to degranulation of mast cells. Each nodule was measured in three perpendicular directions $(a, b, c)$ and the tumor volume was calculated using the formula: $\mathrm{V}=\mathrm{a} \times \mathrm{b} \times \mathrm{c} \times \pi / 6$. A sterile solution of plasmid was injected into the tumor with a $1 \mathrm{ml}$ syringe and $22 \mathrm{G}$ needle. The dose of intratumorally injected plasmid ranged from 0.5 to $1 \mathrm{mg} / \mathrm{cm}^{3}$ of tumor tissue per one EGT session for tumors with volumes ranging from $0.1 \mathrm{~cm}^{3}$ to 2.5 $\mathrm{cm}^{3}$. In smaller tumors $\left(<0.1 \mathrm{~cm}^{3}\right)$ and in larger tumors $\left(>2.5 \mathrm{~cm}^{3}\right)$, an arbitrary dose per tumor nodule was set, being $0.1 \mathrm{mg}$ for smaller and $1 \mathrm{mg}$ for larger tumors (Table 2). Ten minutes after plasmid injection, electric pulses were delivered using the electric pulse generator Cliniporator ${ }^{\mathrm{TM}}$ (IGEA s.r.l., Carpi, Italy), using needle electrodes (2 arrays each composed of 4 electrodes with a 4 -mm distance between them). One high voltage pulse was delivered $(1 \times 1200 \mathrm{~V} / \mathrm{cm}, 100 \mu \mathrm{s})$, immediately followed by 8 low voltage pulses $(8 \times 50 \mathrm{~ms}, 140 \mathrm{~V} / \mathrm{cm}, 2 \mathrm{~Hz})$. After the electrogene procedure, all dogs received single intravenous application of carprofene (Rimadyl, Pfizer Animal Health, Dundee, United Kingdom; $4 \mathrm{mg} / \mathrm{kg}$ of bodyweight). When they fully recovered from anesthesia, they were released from hospital. Prior to release into the home environment, animals received Elizabethan collars in order to prevent any wound licking. Furthermore, treated tumor nodules were protected with suitable dressing to prevent any possible contact of humans or animals with the electroporated area.

\section{Evaluation of response to therapy and possible side effects}

Animals were examined one, two and four weeks after each EGT session and thereafter monthly. At each examination, a local as well as systemic response to the therapy was determined, along with observation for possible side effects.

The local response to therapy was evaluated with repeated measurements of tumor size as described above and calculation of tumor volumes. Additionally, in animals which underwent surgical excision of tumors, histological examination of tumor samples was performed. The systemic response to the treatment was assessed by determination of IL-12 and IFN- $\gamma$ in patients' sera.

The possible occurrence of local or systemic side effects was evaluated at each follow- up with clinical examination of the animals and careful as- 
sessment of the appearance of the electroporated area for any possible clinical signs, including erythema, oedema, pain, secretions, necrosis, etc. Furthermore, blood samples were taken for the same bloodwork as before the procedure.

\section{Statistical analysis}

Statistical analysis was performed using SigmaPlot software (Systat Software, Inc., Richmond CA, USA). To determine the significance of differences in tumor volumes of MCT before and after the treatment, a Friedman repeated Measures Analysis of Variance on Ranks was performed. Values of $\mathrm{P}<0.05$ were considered significant.

\section{Results}

\section{Response to the therapy}

Before EGT, tumor volumes ranged from 0.03 to $25.4 \mathrm{~cm}^{3}$. Treated nodules reached the smallest volume one to two weeks after the last EGT procedure (Table 2), with their volumes ranging from 0.005 to $18 \mathrm{~cm}^{3}$, which was statistically significantly smaller compared to the volumes before EGT. One week after the last EGT session and before induction of any other therapeutic procedure, the tumor volume was reduced in 9/11 treated tumors, it had not changed in 1/11 treated tumors and progressed in $1 / 11$ treated tumors. In nodules where reduction of tumor volume was achieved, it ranged from 13\% and up to $83 \%$ of the initial value (median $50 \%$ ).

In two patients (\#1 and \#6) with a total of three tumor nodules, EGT with IL-12 was not followed by any other treatment (Table 2). In both patients, the tumor nodules reduced in size and treated patients responded to therapy with stable disease throughout the very long observation period (36 and 44 months).

Two patients (\#3 and \#8) received systemic chemotherapy with CCNU (Table 2). In one of these patients (\#3) with stage II disease (regional lymph node involvement), stable disease with reduction in tumor volume by $50 \%$ was achieved with regression of detectable mast cell infiltration of lymph nodes and without any signs of distant metastases throughout the 12-month observation period. The second patient, (\#8), treated with a combination of EGT and systemic chemotherapy had recurrent stage III disease, unresponsive to any treatment and was euthanized 5 months after EGT due to progress of the disease with systemic clinical signs.
In one patient (\#4) with two tumor nodules, EGT in one nodule was followed by surgical removal of the tumor and in the other, due to its location in the perineum, EGT was followed by ECT, achieving a complete response in the treated nodule (Table 2).

In four patients (\#2, \#4, \#5 and \#7), surgical removal of 4 grade II and one grade III tumor nodules was performed one week post-EGT (Table 2). After surgery, three had a complete response to therapy without any signs of local recurrence or metastatic disease in over a 1-year observation period. The remaining one (\#7), with stage III disease, had progression of clinical signs and was euthanized 2 months after EGT.

\section{Histology of the tumors}

All surgically removed tumors underwent histological examination. The control group represented MCT samples taken with a biopsy from the same tumor nodule during the diagnostic workup before inclusion into the clinical study or from untreated tumor nodules which were simultaneously removed in patients with multiple nodules.

Histological analysis of MCT prior to IL-12 EGT showed nonencapsulated dermal and/or subcutaneous infiltrative growing masses composed of round cells arranged in densely packed cords. Most malignant mast cells were recognized in H\&E stained slides by their cytoplasmic light gray-blue granules. Granules stained metachromatically with cationic dyes (toluidine blue staining). Most cells had a single nucleus, however same binucleated mast cells were also found. Among mast cell cords, variable numbers of diffusely distributed or aggregated eosinophils were seen. After the treatment, the distributions of viable malignant mast cells were reduced in comparison to the pretreatment samples (Figure 1A, B). Instead of mast cells in the dermis and subcutis, clusters of leukocyte infiltration were found. Large areas of mostly lymphocytes and plasma cells with eosinophilic cytoplasm and perinuclear halos were seen (Figure 1C, D). No similar infiltrates were found in samples of untreated lesions. Among immune cells, some degranulated mast cells were noticed without prominent neutrophils or eosinophils.

\section{Hematology and systemic release of cytokines}

In order to evaluate any possible systemic effects of the therapy, serum concentrations of human IL-12 


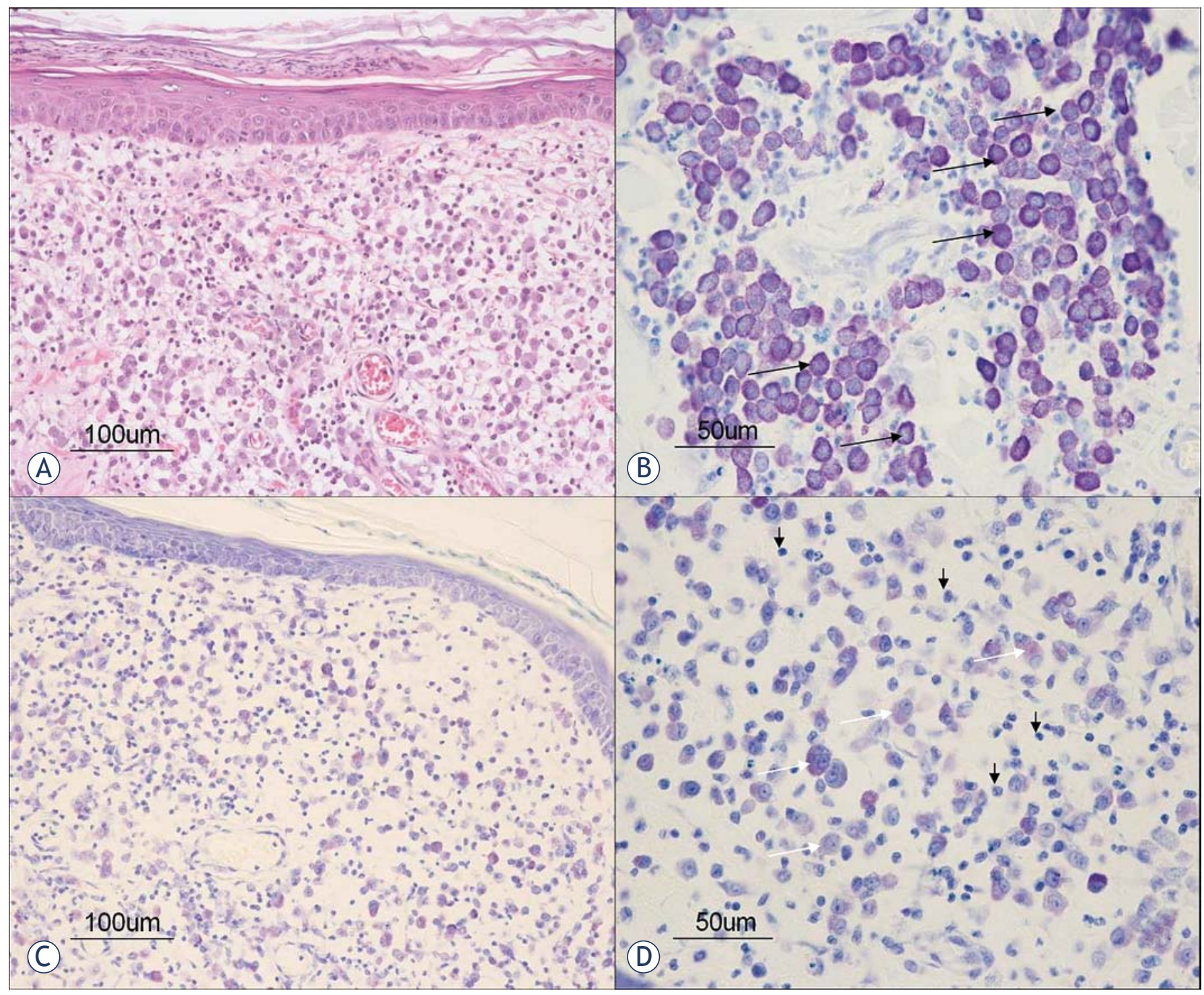

FIGURE 1. Histological pictures of MCTs before (A and B) and after (C and D) EGT. A. Tumor mast cells are loosely arranged in the dermis without epidermal invasion (haematoxylin and eosin staining). B. Tumor mast cells (arrow) have a well granulated metachromatic cytoplasm (toluidine blue staining). C. Decreased number of mast cells in the dermis after the treatement (toluidine blue staining). D. Note the degranulated tumor mast cells with metachromatically weakly stained cytoplasm (white arrows) intermingled with numerous inflammatory cells (black arrows) (toluidine blue staining).

and canine IFN- $\gamma$ were measured at regular timepoints after the procedure.

IL-12 was detected in 5 samples from 3 patients, with serum concentrations ranging from 1 to 12.2 $\mathrm{pg} / \mathrm{ml}$. IFN- $\gamma$ was detected in 5 samples from 2 patients, with concentrations ranging from 123.0 to $815.6 \mathrm{pg} / \mathrm{ml}$. IL-12 and/or IFN- $\gamma$ were detected in a total of 4 patients. All positive samples were collected after the EGT procedure; in samples, taken before the procedure, neither of the cytokines was detected. The highest systemic concentrations of transgene products were detected in patient \#3. In this dog, all 3 post-EGT samples were positive for both IL-12 (2.3 pg/ml to $12.2 \mathrm{pg} / \mathrm{ml})$ and IFN- $\gamma(170$ $\mathrm{pg} / \mathrm{ml}$ to $388.1 \mathrm{pg} / \mathrm{ml}$ ) (Table 3).

In order to evaluate possible side effects of the procedure, clinical examinations of patients were carried out on a regular basis, with careful examination of the electroporated area and selected bloodwork.

We did not detect any evident side effects, either locally or systemically. All patients, which responded to treatment remained in good clinical condition throughout the observation period, without any additional clinical signs of disease. All analyzed blood parameters remained within 
Table 3. Haematology and biochemistry profile of patient No. 3 with the highest number of serum samples, positive to IL-12 and IFN-Y

\begin{tabular}{|c|c|c|c|c|c|c|}
\hline & & DAY 0 & DAY 7 & DAY 14 & DAY 28 & REFERENCE VALUES \\
\hline \multirow[t]{2}{*}{ Cytokine conc. } & IL-12 (pg/ml) & 0 & 2.3 & 6.1 & 12.2 & N/A \\
\hline & IFN-Y (pg/ml) & 0 & 388.1 & 179.6 & 164.3 & N/A \\
\hline \multirow{2}{*}{ Haematology } & $\mathrm{RBC}\left(\times 10^{12} / \mathrm{l}\right)$ & 5.41 & 5.38 & 8.1 & 7.97 & $5.5-8.5$ \\
\hline & $\mathrm{HCT}(\mathrm{L} / \mathrm{L})$ & 0.38 & 0.36 & 0.58 & 0.57 & $0.37-0.55$ \\
\hline \multirow[t]{4}{*}{ Biochemistry panel } & BUN (mmol/l) & 3.28 & 4.2 & 3.94 & $N / D$ & $2.5-9.6$ \\
\hline & Crea $(\mu \mathrm{mol} / \mathrm{l})$ & 74.54 & 75.7 & 71.23 & N/D & $44.2-132.6$ \\
\hline & SAP (U/I) & 47.3 & 27.5 & 23.8 & 47.1 & $20-156$ \\
\hline & ALT (U/I) & 21.8 & 64.0 & 58.6 & 217.6 & $21-102$ \\
\hline
\end{tabular}

WBC, white blood cells; RBC, red blood cells; HCT, haematocrit; PLT, platelets; BUN, blood urea nitrogen, Crea, creatinine; SAP, serum alkaline phosphatase; ALT, alanin aminotransferase; N/D, not detected

the normal reference range. The few alterations in bloodwork parameters which occurred were only minimal and clinically irrelevant (for example, mild haemoconcentration in two samples). In two patients, slight elevations of SAP and/or ALT were detected in samples, obtained 1 and 2 months after the procedure, but they were considered side effects of CCNU chemotherapy, which was started 2 weeks after EGT. One of these two patients was euthanized due to progressive disease, unresponsive to all therapies whilst in the other, serum concentrations of both SAP and ALT returned within the reference range after discontinuation of chemotherapy. Details of hematology and the biochemistry profile in patient \# 3 , in which the best systemic response to treatment with the highest number of serum samples positive to IL-12 and IFN- $\gamma$ was achieved, are summarized in Table 3.

\section{Discussion}

Our study demonstrates that intratumoral EGT with IL-12 in canine MCT elicits good local antitumor effects in treated animals without any noticeable side effects. Local antitumor effects of this therapy can be seen as significant reduction in tumor size (median reduction of the pretreatment tumor volumes was $50 \%$ ) and change in histological structure with reduction in the number of malignant mast cells coupled with infiltration of inflammatory cells in treated tumors. We also demonstrated that systemic release of the transgene product is possible after intratumoral EGT.

EGT is a novel treatment in medicine which has already entered the clinical stage of research in hu- man oncology ${ }^{20}$, and is also gaining some interest in veterinary medicine. ${ }^{11-15,25}$ The results of EGT with various therapeutic genes including plasmid encoding IL-12 in the treatment of tumors are promising..$^{21}$ In a recent human clinical study, EGT with $I L-12$ plasmid in the treatment of melanoma patients showed local as well as systemic antitumor effects with regression of tumor nodules and with minimal systemic toxicity. ${ }^{20}$

In our study, we treated spontaneously occurring cutaneous nodules of MCT in eight canine patients utilizing locally applied EGT with a therapeutic gene encoding human IL-12. The majority of treated nodules regressed in size after the EGT procedure by $50 \%$ (median) around 1-2 weeks after the last EGT session. These results can be compared to published results by Rakhmilevich et al. on growth delay of murine P815 mastocytoma ${ }^{26}$ after bioballistic $I L-12$ gene therapy and Heinzerling et al. ${ }^{17}$, who utilized a similar approach for treatment of melanoma in horses with direct intratumoral application of plasmid DNA encoding human IL-12 without subsequent electroporation. In the murine mastocytoma model, a $60 \%$ reduction in volume was achieved three weeks after therapy. In equine melanomas, the mean reduction in size was $69 \%$ of the initial volume. It is possible that in dogs more repetitions of treatment sessions would result in even more pronounced tumor regression, since in the murine MCT model 6 repetitions whilst in horses up to 3 repetitions of treatment were necessary to reach a significant reduction in tumor size.

Histological analysis revealed a noticeable change in tumor morphology after EGT with IL-12. Beside reduction in the number of malignant mast cells, the most prominent feature of treated tumors 
was diffuse infiltration of tumor tissue with lymphocytes and plasma cells as well as degranulation of remaining mast cells. These histological changes are in accordance with other studies employing intratumoral IL-12 gene therapy with different delivery systems, where intra- and peritumoral lymphocytic infiltration was found to be a major contribution to histological changes in treated nodules. ${ }^{17,20,27}$ The importance of lymphocytic infiltration of treated tumors after intratumoral IL-12 EGT was shown in a variety of preclinical studies. ${ }^{27,28}$ It was established that this mode of therapy does not elicit any antitumor effect in athymic mice deficient in $\mathrm{T}$ cells in contrast to immunocompetent mice, indicating the vital role of $\mathrm{T}$ lymphocytes in the antitumor activity of local IL-12 EGT. ${ }^{27,28}$ In our study, intratumoral IL-12 EGT resulted in an immunological response with lymphocytic infiltration of treated tumors, which can be further indication that plasmid encoding human IL-12 is biologically active in dogs in vivo, as it was proposed to be in in vitro settings. ${ }^{24}$

The importance of the systemic action of IL-12 after local delivery in addition to local intratumoral accumulation of IL-12 has already been shown, demonstrating that circulating IL-12 is responsible for systemic antitumor effects, e.g. an antitumor effect on distant untreated tumors and prevention of distant metastases..$^{29-32}$ Therefore, systemic release of the transgene product would be extremely favorable in clinical settings, expanding local antitumor therapy into systemic treatment. In our study, systemic release of human IL-12 was detected in only three patients. Even though at the preclinical level there is contradictory data on the possibility of systemic transgene release after intratumoral $I L-12$ EGT, in two studies on induced transmissible veneral tumors in $\operatorname{dog} s^{14,15}$, similar concentrations of human IL-12 as in our three patients were detected. Therefore, further investigations are warranted to determine conditions for achieving systemic effects of intratumoral EGT with $I L-12$ in dogs, since some release of IL-12 from treated tumors is clearly possible.

In treated patients we paid attention to two possible groups of adverse side effects. IL-12-based intratumoral EGT could lead to degranulation of mast cells, causing histamine release from granules, which is one of the major concerns of any mechanical manipulation of MCT. It can result in either local side effects, demonstrated as peritumoral swelling, edema and erythema, or in systemic clini- cal signs, for example, gastrointestinal ulceration, or even life-threatening hypotension, arrhythmias and bronchospasm. We did not observe any of these side effects, even though moderate mechanical manipulation of tumors could not be avoided since penetrating needle electrodes, which had to be inserted intratumorally, were used for EGT.

The second group of possible side effects is connected to systemic IL-12 toxicity. It has been shown that systemic administration of recombinant protein IL-12 is associated with multiple serious adverse side effects, including renal and systemic toxicity. High-dose levels were also linked to temporary immune suppression, which would not be favorable for effective immunotherapy. ${ }^{33}$ Local intratumoral IL-12 EGT was associated with significantly less adverse effects, while exhibiting a pronounced antitumor effect, as demonstrated by Heller et al. on a mouse melanoma model. ${ }^{34}$ Even so, monitoring renal function with selected laboratory parameters (e.g. serum concentrations of BUN, creatinine) was recommended in any IL-12-based therapy. ${ }^{34}$ In our study, all monitored hematological and biochemical parameters in blood samples remained within reference limits throughout the whole observation period with only few transient clinically nonsignificant and nonspecific alterations, which could be attributed to other factors. The clinical status of all animals that responded to therapy remained unaltered and they didn't show any changes in appetite, water intake and general behavior.

In conclusion, the results of our study demonstrate that intratumoral IL-12 EGT in canine MCT is a feasible, simple and safe therapeutic procedure, which exerts local transgene expression with systemic release of the encoded protein, making it a promising treatment for canine patients with MCT. However, further refinement for effective use of this method in treatment of MCT is needed, with emphasis on optimization of the treatment proto$\mathrm{col}$, including determination of appropriate dosage of the plasmid used, as well as the best possible number of EGT repetitions and optimal time interval between them.

\section{Acknowledgements}

The authors acknowledge the financial support of the state budget by the Slovenian Research Agency (Projects No. P3-0003, P4-0053 and J3-2277). 


\section{References}

1. Thamm DH, Vail DM. Mast Cell Tumors. In: Withrow SJ, Wail DM, editors. Withrow and MacEwen's small animal clinical oncology, 4th edn. St. Louis: Saunders; 2007. p. 402-16.

2. Maureen C, XinRu T, Benett P. Combination CCNU and Vinblastine chemotherapy for canine mast cell tumors: 57 cases. Vet Comp Onc 2009; 7: 196206

3. Cemazar M, Tamzali Y, Sersa G, Tozon N. Mir LM, Miklavcic D, et al. Electrochemotherapy in veterinary oncology. J Vet Intern Med 2008; 22 826-31.

4. Mir LM, Glass LF, Sersa G, Teissie J, Domenge C, Miklavcic D, et al. Effective treatment of cutaneous and subcutaneous malignant tumors by electrochemotherapy. Br J Cancer 1998; 77: 2336-42.

5. Sersa G, Miklavcic D, Cemazar M, Rudolf Z, Pucihar G, Snoj M. Electrochemotherapy in treatment of tumors. Eur J Surg Oncol 2008; 34 232-40.

6. Gothelf A, Mir LM, Gehl J. Electrochemotherapy: results of cancer treatment using enhanced delivery of bleomycin by electroporation. Cancer Treat Rev 2003; 29: 371-87.

7. Neumann E, Schaefer-Ridder M, Wang Y, Hofschneider PH. Gene transfer into mouse lyoma cells by electroporation in high electric fields. EMBO J 1982; 1: 841-5.

8. Mir LM. Nucleic acids electrotransfer-based gene therapy (electrogenetherapy): past, current, and future. Mol Biotechnol 2009; 43: 167-76.

9. Cemazar M, Golzio M, Sersa G, Rols MP, Teissié J. Electrically-assisted nucleic acids delivery to tissues in vivo: where do we stand? Curr Pharm Des 2006; 12: 3817-25.

10. Heller LC, Heller R. In vivo electroporation for gene therapy. Hum Gene Ther 2006; 17: 890-7.

11. Fewell JG, MacLaughlin F, Mehta V, Gondo M, Nicol F, Wilson E, et al. Gene therapy for the treatment of hemophilia B using PINC-formulated plasmid delivered to muscle with electroporation. Mol Ther 2001; 3: 574-83.

12. Draghia-Akli R, Hahn KA, King GK, Cummings KK, Carpenter RH. Effects of plasmid-mediated growth hormone-releasing hormone in severely debilitated dogs with cancer. Mol Ther 2002; 6: 830-6.

13. Pavlin D, Tozon N, Sersa G, Pogacnik A, Cemazar M. Efficient electrotransfection into canine muscle. Technol Cancer Res Treat 2008; 7: 45-54.

14. Chou PC, Chuang TF, Jan TR, Gion HC, Huang YC, Lei HJ, et al. Effects of immunotherapy of IL-6 and IL-5 plasmids on transmissible veneral tumor in beagles. Vet Immunol Immunopathol 2009; 130: 25-34.

15. Chuang, TF, Lee SC, Liao KW, Hsiao YW, Lo $\mathrm{CH}$, Chiang BL, et al. Electroporation-mediated IL-12 gene therapy in a transplantable canine cancer model. Int J Cancer 2009; 125: 698-707.

16. DelVecchio M, Bajetta E, Canova S, Lotze MT, Wesa A, Parmiani G, et al. Interleukin-12: biological properties and clinical application. Clin Cancer Res 2007; 13: 4677-85.

17. Heinzerling LM, Feige K, Rieder S, Akens MK, Dummer R, Stranzinger G, et al. Tumor regression induced by intratumoral injection of DNA coding for human interleukin 12 into melanoma metastases in gray horses. J Mol Med 2001; 78: 692-702

18. Siddiqui F, Li C-Y, LaRue SM, Poulson JM, Avery PR, Pruitt AF, et al. A phase I trial of hyperthermia-induced interleukin-12 gene therapy in spontaneously arising feline soft tissue sarcomas. Mol Cancer Ther 2007; 6: 380-9.

19. Kamensek U, Sersa G. Targeted gene therapy in radiotherapy. Radiol Oncol 2008; 42: 115-35.

20. Daud Al, DeConti RC, Andrews S, Urbas P, Riker Al, Sondak VK, et al. Phase I trial of interleukin-12 plasmid electroporation in patients with metastatic melanoma. J Clin Oncol 2008; 26: 5896-903.

21. Cemazar M, Jarm T, Sersa G. Cancer Electrogene Therapy with Interleukin-12. Cur Gene Ther 2010; 10: 300-11.

22. Patnaik AK, Ehler WY, MacEwen EG. Canine cutaneous mast cell tumors: morphological grading and survival in 83 dogs. Vet Pathol 1984; 21: 469-74.
23. Buettner M, Belke-Louis G, Rziha HJ, Mclnnes C, Kaaden OR. Detection, cDNA cloning and sequencing of canine interleukin 12. Cytokine 1998; 10: 241-8.

24. Phillips BS, Padilla ML, Dickerson EB, Lindstrom MJ, Helfand SC Immunostimulatory effects of human recombinant interleukin-12 on peripheral blood mononuclear cells from normal dogs. Vet Immunol Immunopathol 1999; 70: 189-201.

25. Tamzali Y, Couderc B, Rols MP, Golzio M, Teissie J. Equine cutaneous tumors treatment by electro-chemo-immuno-therapy. In: Jarm T, Kramar P, Zupanic A, editors. IFBME proceedings, vol 16. New York: Springer; 2007. p. 610-3.

26. Rakhmilevich AL, Timmins JG, Janssen K, Pohlmann EL, Sheehy MJ, Yang NS. Gene gun-mediated IL-12 gene therapy induces antitumor effects in the absence of toxicity: a direct comparison with systemic IL-12 protein therapy. J Immunother 1999; 22: 135-44.

27. Li S, Zhang L, Torrero M, Cannon M, Barret R. Administration route- and immune cell activation-dependant tumor eradication by IL-12 electrotransfer. Mol Ther 2005; 12: 942-9.

28. Lucas ML, Heller L, Coppola D, Heller R. IL-12 plasmid delivery by in vivo electroporation for the successful treatment of established subcutaneous B16.F10 melanoma. Mol Ther 2002; 5: 668-75.

29. Pavlin D, Cemazar M, Kamensek U, Tozon N, Pogacnik A, Sersa G. Local and systemic antitumor effect of intratumoral and peritumoral IL-12 electrogene therapy on murine sarcoma. Cancer Biol Ther 2009; 8: 2114-22.

30. Tevz G, Kranjc S, Cemazar M, Kamensek U, Coer A, Krizan M, et al. Controlled systemic release of interleukin-12 after gene electrotransfer to muscle for cancer gene therapy alone or in combination with ionizing radiation in murine sarcomas. J Gene Med 2009; 11: 1125-37.

31. Lucas ML, Heller R. IL-12 gene therapy using an electrically mediated nonviral approach reduces metastatic growth of melanoma. DNA Cell Biol 2003; 22: $755-63$.

32. Lee SC, Wu CJ, Wu PY, Huang YL, Wu CW, Tao MH. Inhibition of established subcutaneous and metastatic murine tumors by intramuscular electroporation of the interleukin-12 gene. J Biomed Sci 2003; 10: 73-86.

33. Leonard JP, Sherman ML, Fisher GL, Buchanan L, Larsen G, Atkins MB, et al Effects of single dose interleukin 12 exposure on interleukin-12 associated toxicity and interferon-gamma production. Blood 1997; 90: 2541-8.

34. Heller L, Merkler K, Westover J, Cruz Y, Coppola D, Benson K, et al. Evaluation of toxicity following electrically mediated interleukin-12 gene delivery in a B16 mouse melanoma model. Clin Cancer Res 2006; 12: 3177 83. 